\title{
Anwendung der
}

D-A-CH-Referenzwerte für die Nährstoffzufuhr

\section{Grundlage für die Umsetzung einer vollwertigen Ernährung gesunder Menschen}

Margrit Richter, Angela Bechthold

Eine ausgewogene, den Bedarf an Nährstoffen und Energie deckende (isoenergetische)

Ernährung ist eine wichtige Grundlage für Wachstum und Entwicklung sowie für den langfristigen Erhalt von Gesundheit, Leistungsfähigkeit und Wohlbefinden des Menschen. Die D-A-CH-Referenzwerte benennen Mengen für die Nährstoff- und Energiezufuhr gesunder Menschen.

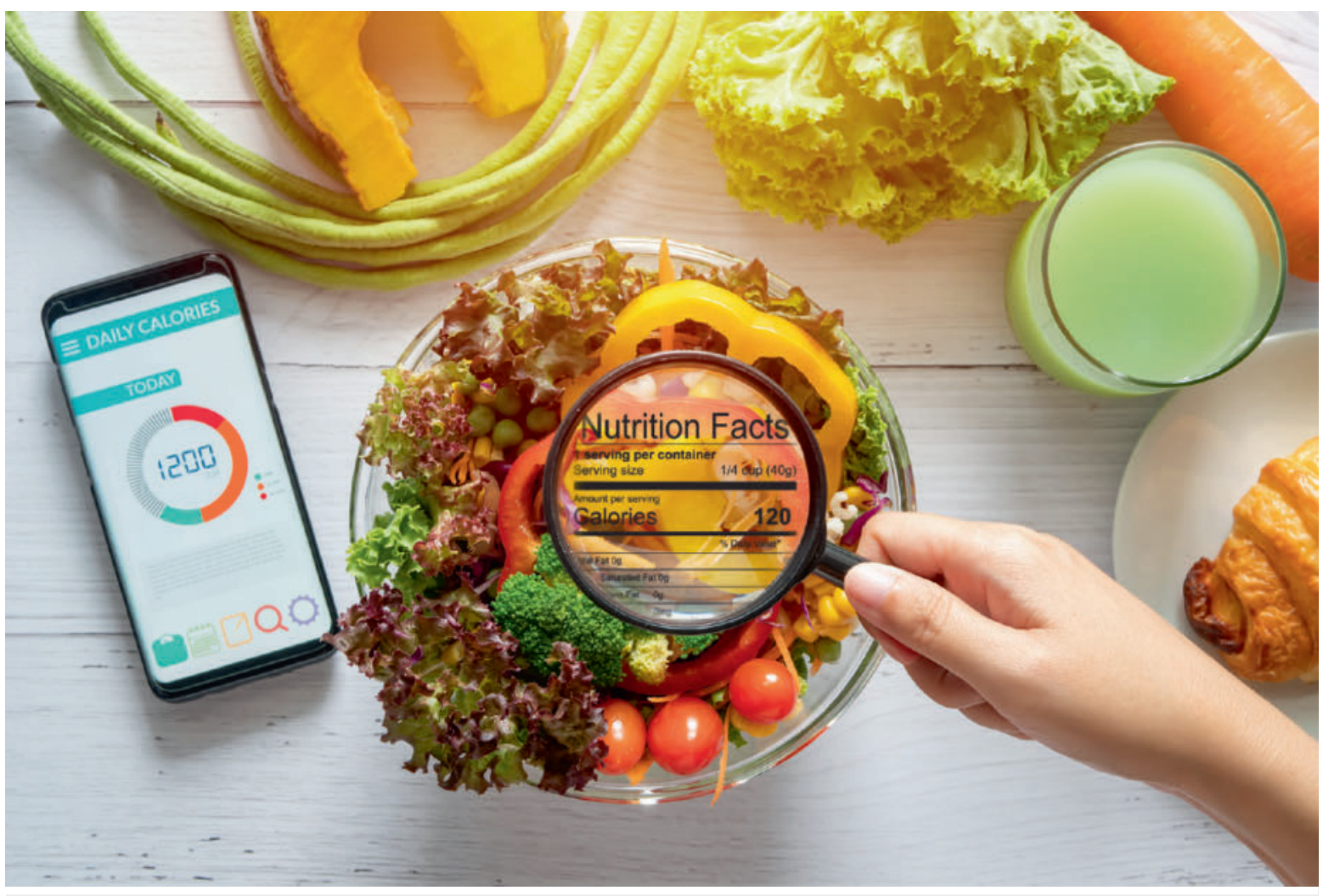

Wie viel ist notwendig? Referenzwerte bieten eine wissenschaftlich fundierte Grundlage für eine sichere Nährstoffzufuhr gesunder Menschen. Foto: asiandelight/Adobe Stock 
Die Deutsche Gesellschaft für Ernährung e. V. (DGE) veröffentlicht gemeinsam mit der Österreichischen (ÖGE) und der Schweizerischen (SGE) Gesellschaft für Ernährung die D-A-CH-Referenzwerte für die Nährstoffzufuhr [1]. Diese benennen Mengen für die Zufuhr von Energie und Nährstoffen für gesunde Menschen. Personen mit Krankheiten, bestehendem Nährstoffmangel oder solche, die durch Genussmittel oder eine regelmäßige Medikamenteneinnahme belastet sind, müssen individuell ernährungsmedizinisch beraten und betreut werden.

Referenzwerte werden für Nährstoffe, Wasser, Ballaststoffe sowie für Energie ausgesprochen. Zu den energieliefernden Nährstoffen gehören Kohlenhydrate, Fett und Protein. Ein internationaler Vergleich der D-A-CH-Referenzwerte für diese Nährstoffe ist im nachfolgenden Beitrag von Suchorski et al. in diesem Heft zu finden (S. 174).

\section{D-A-CH-Referenzwerte für die Nährstoffzufuhr}

Je nach wissenschaftlicher Datenlage und physiologischer Rolle sprechen die 3 Gesellschaften im Konsens eine empfohlene Zufuhr, einen Schätzwert oder einen Richtwert aus.

\section{Merke \\ Die empfohlene Zufuhr ist der Referenzwert mit der höchsten Aussagekraft.}

Die empfohlene Zufuhr soll eine bedarfsdeckende Zufuhr für nahezu alle gesunden Individuen einer definierten Personengruppe (Alter, Geschlecht) sichern.

\section{DEFINITION}

Die empfohlene Zufuhr ist die durchschnittliche tägliche Nährstoffzufuhr, die ausreicht, um den Bedarf nahezu aller gesunden Individuen (97,5\%) einer definierten Personengruppe (Geschlecht, Alter) zu decken.

Ausgangspunkt für die Ableitung einer empfohlenen Zufuhr ist die Bestimmung des Bedarfs. Der Bedarf ist die Menge eines Nährstoffs bzw. die Menge an Energie, die gebraucht wird, um den Stoffwechsel und die Funktionen des Organismus aufrechtzuerhalten und die bereits die Bioverfügbarkeit berücksichtigt.

Der Energie- und Nährstoffbedarf ist allerdings von Mensch zu Mensch und von Tag zu Tag verschieden und abhängig von vielen endogenen (z. B. Alter, Geschlecht, Ernährungsstatus, Gesundheitszustand) und exogenen Einflüssen (z. B. Klima, körperliche Aktivität in Beruf und Freizeit). Daher wird als Grundlage zur Ableitung der experimentell ermittelte durchschnittliche Bedarf verwendet. Bei diesem handelt es sich um die tägliche Nährstoffzufuhr, von der angenommen wird, dass sie den Bedarf von $50 \%$ der Menschen einer definierten Personengruppe deckt. Zur Ableitung der empfohlenen Zufuhr wird der durchschnittliche Bedarf gewöhnlich um die zweifache Standardabweichung bzw. den zweifachen Variationskoeffizienten (Streuung: 10-15\%; Zuschlag von 20-30\%) erhöht. Eine empfohlene Zufuhr wird z. B. für Protein in den meisten Altersgruppen auf Grundlage von Stickstoffbilanzstudien ausgesprochen.

Bei einigen Nährstoffen bestehen Unsicherheiten bei der Bestimmung des durchschnittlichen Bedarfs, z. B. aufgrund von Schwankungen der Messwerte oder zu wenigen (geeigneten) Ergebnissen von Untersuchungen am Menschen. In diesen Fällen kann keine empfohlene Zufuhr abgeleitet werden, dann werden Schätzwerte angegeben.

\section{DEFINITION}

Ein Schätzwert wird abgeleitet, wenn der durchschnittliche Bedarf nicht mit wünschenswerter Genauigkeit angegeben werden kann. Er gibt Hinweise auf eine angemessene und gesundheitlich unbedenkliche Zufuhr.

Schätzwerte basieren in der Regel auf einer bei Gesunden beobachteten, aus dem Verzehr abgeleiteten oder experimentell ermittelten Nährstoffzufuhr einer definierten Bevölkerungsgruppe. Ein Beispiel hierfür ist der D-A-CH-Referenzwert für die Proteinzufuhr für Erwachsene ab 65 Jahren. Für diese Altersgruppe lässt sich der Proteinbedarf nicht mit wünschenswerter Genauigkeit aus Stickstoffbilanzstudien bestimmen. Für Erwachsene ab 65 Jahren wird daher ein Schätzwert für eine angemessene Proteinzufuhr angegeben. Ein weiteres Beispiel sind die D-A-CHReferenzwerte für die Nährstoffzufuhr für Säuglinge. Diese werden in der Regel anhand des Nährstoffgehalts der Frauenmilch abgeleitet.

Für Nährstoffe, die für den Organismus nicht essenziell sind und für die daher kein Bedarf besteht, werden Richtwerte ausgesprochen. Darüber hinaus werden Richtwerte angegeben, wenn zwar ein Bedarf besteht, wie für Energie, dieser aber in Abhängigkeit von zahlreichen Einflussfaktoren (z. B. Lebensstil, Beruf) sehr stark variiert. Bei der Ableitung der Richtwerte werden präventive Aspekte dieser Nährstoffe berücksichtigt. Richtwerte haben unter den Referenzwerten die niedrigste Aussagekraft, sie dienen als Orientierungshilfen.

\footnotetext{
Merke

Ein Richtwert ist eine Orientierungshilfe, die aus ernährungsphysiologischer Sicht wünschenswerte Bereiche oder Werte angibt.
} 
Da für Fett und Kohlenhydrate als solche kein durchschnittlicher Bedarf ermittelt werden kann, werden in den D-A-CH-Referenzwerten für die Nährstoffzufuhr für Fett und Kohlenhydrate Richtwerte angegeben.

\section{Anwendung der D-A-CH-Referenzwerte für die Nährstoffzufuhr Anwendungsbereiche}

Die D-A-CH-Referenzwerte für die Nährstoffzufuhr dienen z. B. als eine Grundlage für die Ableitung der lebensmittelbezogenen Ernährungsempfehlungen der DGE:

- 10 Regeln der DGE

- DGE-Ernährungskreis

- Dreidimensionale DGE-Lebensmittelpyramide.

Sie finden in der Praxis unter anderem Einsatz bei der Planung einer vollwertigen Ernährung und bei der Beurteilung der Nährstoffzufuhr. So liegen sie z. B. auch den DGEQualitätsstandards für die Gemeinschaftsverpflegung zugrunde. Auch werden die Referenzwerte als Grundlage für die individuelle Ernährungsberatung genutzt. Dazu ist anzumerken, dass Speisepläne auf der Basis der Referenzwerte bei Einzelpersonen nur zu einer angenäherten Bedarfsdeckung führen können. Mit dem Anspruch absoluter Genauigkeit ist die Planung einer bedarfsdeckenden Ernährung von Einzelpersonen nicht möglich, da der individuelle Bedarf nicht bekannt ist. Auch die exakte Beurteilung des Versorgungszustands einer Einzelperson allein auf Basis der berechneten Nährstoffzufuhr ist aus diesem Grund nicht möglich. Es lässt sich jedoch abschätzen, ob die Nährstoffzufuhr der empfohlenen Zufuhr gerecht wird und somit die oder der Einzelne mit großer Wahrscheinlichkeit adäquat versorgt ist.

\section{Handhabung}

Die den D-A-CH-Referenzwerten zugrunde liegenden Daten sind unterschiedlichen Ursprungs. Daher haben empfohlene Zufuhr, Schätzwert und Richtwert eine unterschiedliche Aussagekraft. Dies ist für eine korrekte Handhabung der Referenzwerte zu berücksichtigen.

Die einzelnen Referenzwerte sind für den Mittelwert der jeweiligen Altersgruppe berechnet und beziehen sich auf die tägliche Zufuhr pro Person. Zudem werden unterschiedliche Verfahren zur Bestimmung des Bedarfs und zur Ableitung von Werten für die empfohlene Zufuhr und Schätzwerten eingesetzt. Daher sollte ein „überscharfes“ Rechnen mit den Referenzwerten vermieden werden. Dies gilt insbesondere für die Unterschiede bei aufeinanderfolgenden Altersgruppen oder zwischen männlichen und weiblichen Personen. Die Einflüsse von Lebensmittelkombinationen, Mahlzeiten oder auch Genussmitteln beispielsweise auf die Absorption sowie den Stoffwechsel bestimmter Nährstoffe können größer sein als die Unterschiede zwischen den empfohlenen Zufuhrmengen für die zuvor genannten Gruppen.
PRAXISTIPP

Die Referenzwerte können und müssen nicht an jedem einzelnen Tag und nicht anteilig durch eine einzelne Mahlzeit erfüllt werden. Es reicht aus, wenn die Vorgaben im Durchschnitt einer Woche erreicht werden.

Da die Referenzwerte die Menge des Nährstoffs angeben, die der menschliche Organismus für Gesundheit, Leistungsfähigkeit und Wohlbefinden benötigt, müssen bei der Planung und Beurteilung der Nährstoffversorgung die Mengen von Nährstoffen berücksichtigt werden, die zum Zeitpunkt des Verzehrs im Lebensmittel noch vorhanden sind (z. B. roh versus gegart).

\section{Unter- bzw. Überschreiten der D-A-CH-Referenzwerte}

Auf die Einzelperson angewandt, ist die empfohlene Zufuhr eine Zielgröße, um die ausreichende Zufuhr des jeweiligen Nährstoffs annähernd sicherzustellen.

Merke

Bei einer Nährstoffzufuhr in Höhe der empfohlenen Zufuhr ist die betreffende Person mit großer Wahrscheinlichkeit adäquat versorgt.

Eine Unterschreitung der empfohlenen Zufuhr („rechnerische Unterversorgung“) erlaubt nicht zwangsläufig den Rückschluss auf einen tatsächlich vorliegenden Mangel. Sie erhöht nur die Wahrscheinlichkeit einer Unterversorgung. Dies gilt ebenso für das Unterschreiten der Schätzwerte.

Bei einer über den Referenzwerten liegenden Zufuhr eines Nährstoffs kann es zu unerwünschten toxischen sowie pharmakologischen Wirkungen kommen. Ab welcher Menge das Risiko für schädliche Nebenwirkungen erhöht ist, unterscheidet sich je nach Nährstoff. Soweit entsprechende Daten vorliegen, werden deshalb von internationalen Fachgesellschaften für einzelne Nährstoffe tolerierbare Gesamtzufuhrmengen (Tolerable Upper Intake Level [UL]) abgeleitet, z. B. von der Europäischen Behörde für Lebensmittelsicherheit (EFSA) [2]. Wird diese tolerierbare Gesamtzufuhrmenge regelmäßig überschritten, steigt das Risiko für das Auftreten von schädlichen Nebenwirkungen.

\section{DEFINITION}

Die tolerierbare Gesamtzufuhrmenge ist die höchste chronische Zufuhrmenge eines Nährstoffs (in der Regel als Summe aus der täglichen Zufuhr mit der Nahrung inklusive angereicherter Lebensmittel und Nährstoffpräparate), die als unwahrscheinlich beurteilt wird, ein Risiko für schädigende Wirkungen auf die Gesundheit darzustellen. 
Für die energieliefernden Nährstoffe gibt es keine Daten zu tolerierbaren Gesamtzufuhrmengen. Die EFSA sieht eine Proteinzufuhr in doppelter Höhe des eigenen Referenzwerts (population reference intake [PRI]: 0,83 g/kg Körpergewicht pro Tag) für Erwachsene als sicher an [3]. Laut World Health Organization (WHO) zeigen Beobachtungen, dass eine Proteinzufuhr in 3- bis 4-facher Höhe des Referenzwerts über einen längeren Zeitraum ohne unerwünschte Symptome möglich ist. Aus diesen Beobachtungen kann jedoch nicht geschlossen werden, dass negative Wirkungen bei einer so hohen Proteinzufuhr generell nicht auftreten [4].

Die Richtwerte für die Zufuhr von Fett und Kohlenhydraten sind als Orientierungshilfen zu verstehen. Die DGE hält ein Unterschreiten des Richtwerts für die Kohlenhydratzufuhr, welcher > 50 EN \% beträgt, für vertretbar, wenn folgende Kriterien erfüllt sind [5]:

- Eine ausreichende Versorgung mit allen unentbehrlichen Nährstoffen (Vitamine, Mineralstoffe, essenzielle Fettsäuren) und mit Ballaststoffen ist sichergestellt.

- Die Getreideballaststoffe haben einen wesentlichen Anteil an der Gesamtballaststoffzufuhr, wobei hauptsächlich Vollkornprodukte verzehrt werden sollen.

- Es kommt nicht zu einer gesteigerten Zufuhr von gesättigten Fettsäuren und trans-Fettsäuren.

- Der zusätzliche Proteinkonsum stammt aus pflanzlichen Lebensmitteln und nicht aus einem erhöhten Fleischverzehr, besonders nicht aus rotem Fleisch.

Die Verringerung des Kohlenhydratanteils in der Ernährung führt zwangsläufig zu einer Erhöhung der Anteile anderer energieliefernder Nährstoffe. Dabei sollte auf die Qualität der Nährstoffe und ihre Quellen geachtet werden. Ein höherer Anteil von Fett an der Gesamtenergiezufuhr sollte aus Pflanzenölen oder Nüssen und Samen stammen, um eine ernährungsphysiologisch günstige Fettsäurenzusammensetzung zu gewährleisten.

Bei einer deutlich über dem Bedarf liegenden Proteinzufuhr sollte auf eine ausreichende Flüssigkeitszufuhr geachtet werden, da der beim Proteinabbau entstehende Harnstoff mit dem Urin ausgeschieden werden muss [3][6]. Bei Personen mit eingeschränkter Nierenfunktion kann eine deutlich über dem Bedarf liegende Proteinzufuhr ein Fortschreiten der Niereninsuffizienz auslösen [7][8].

\section{INFOMATERIAL}

Die D-A-CH-Referenzwerte für die Nährstoffzufuhr sowie weiterführende Informationen zu verschiedenen Nährstoffen sind auf der Homepage der DGE unter www.dge.de/wissenschaft/referenzwerte/ zugänglich.
Der Ringordner „Referenzwerte für die Nährstoffzufuhr“ enthält zu jedem Nährstoff ausführliche Erläuterungen, z. B. Informationen zu Mangelerscheinungen und Überversorgung, zur Versorgungssituation in Deutschland sowie zu den Ableitungen der neuen Referenzwerte.

\section{KERNAUSSAGEN}

Die D-A-CH-Referenzwerte für die Nährstoffzufuhr sind eine Grundlage zur Umsetzung einer vollwertigen Ernährung. Die Zufuhr von Nährstoffen einschließlich Ballaststoffen in Höhe der Referenzwerte kann prinzipiell durch eine abwechslungsreiche Ernährung mit einem hohen Anteil an pflanzlichen Lebensmitteln erfolgen. Wie diese gestaltet werden kann, zeigen die lebensmittelbezogenen Ernährungsempfehlungen der DGE (https://www.dge. de/ernaehrungspraxis/vollwertige-ernaehrung/). Die genaue Ausgestaltung dieser Ernährung kann individuell unterschiedlich sein.

\section{Interessenkonflikt}

Margrit Richter und Angela Bechthold sind Mitarbeiterinnen der Deutschen Gesellschaft für Ernährung e. V. (DGE).

\section{Korrespondierende Autorin}



\section{Dr. Margrit Richter}

Margrit Richter ist promovierte Ökotrophologin und arbeitet als wissenschaftliche Mitarbeiterin im Referat Wissenschaft der Deutschen Gesellschaft für Ernährung e.V. (DGE). Seit 2017 koordiniert sie die Überarbeitung der D-A-CH-Referenzwerte für die Nährstoffzufuhr.

Korrespondenzadresse

\author{
Dr. Margrit Richter \\ Deutsche Gesellschaft für Ernährung \\ Godesberger Allee 18 \\ 53175 Bonn \\ E-Mail: corresponding_author@dge.de
}


[1] Deutsche Gesellschaft für Ernährung, Österreichische Gesellschaft für Ernährung, Schweizerische Gesellschaft für Ernährung, Hrsg. Referenzwerte für die Nährstoffzufuhr. 2. Aufl., 5. aktualisierte Ausgabe. Bonn; 2019

[2] EFSA (European Food Safety Authority), Hrsg. Dietary reference values for nutrients: summary report. EFSA Supporting publication 2017; 14: 1133

[3] EFSA (European Food Safety Authority), Hrsg. Scientific opinion on dietary reference values for protein. EFSA Journal 2012; 10: 2557

[4] WHO (World Health Organization). Protein and amino acid requirements in human nutrition. Genf: WHO; 2007

[5] https://www.dge.de/fileadmin/public/doc/ws/position/ DGE-Positionspapier-Richtwerte-Energiezufuhr-KH-undFett.pdf. Stand: 29.07.2019

[6] Lemon PW. Is increased dietary protein necessary or beneficial for individuals with a physically active lifestyle? Nutr Rev 1996; 54: S169-S175

[7] Fouque D, Laville M. Low protein diets for chronic kidney disease in non diabetic adults. Cochrane Database Syst Rev 2009; CD001892

[8] Knight EL, Stampfer MJ, Hankinson SE et al. The impact of protein intake on renal function decline in women with normal renal function or mild renal insufficiency. Ann Intern Med 2003; 138: 460-467

Bibliografie

DOI https://doi.org/10.1055/a-0926-0750

Ernährung \& Medizin 2019; 34: 169-173

(c) Georg Thieme Verlag KG Stuttgart · New York

ISSN 1439-1635 